\title{
A favorable tumor size to define papillary thyroid microcarcinoma: an analysis of II 76 consecutive cases
}

This article was published in the following Dove Press journal:

Cancer Management and Research

\section{Yanping Gong \\ Genpeng Li \\ Jianyong Lei \\ Jiaying You \\ Ke Jiang \\ Zhihui Li \\ Rixiang Gong \\ Jingqiang Zhu}

Thyroid and Parathyroid Surgery Center, West China Hospital of

Sichuan University, Chengdu, China
Correspondence: Jingqiang Zhu

Thyroid and Parathyroid Surgery

Center, West China Hospital of Sichuan

University, 37 Guoxuexiang Road,

Chengdu 610041, China

$\mathrm{Tel} / \mathrm{fax}+862885423822$

Email zjqdoctor@।63.com
Background: The optimal treatment strategy for papillary thyroid microcarcinoma (PTMC) has remained controversial. The purpose of this study was to provide a new reference value for PTMC to aid the selection of optimal management for minute lesions.

Patients and methods: A pool of 1176 consecutive patients who met the inclusion criteria were ultimately enrolled in this study. The correlation of papillary thyroid carcinoma (PTC) tumor size and lymph node metastasis was analyzed. Receiver operating characteristic curve studies were conducted to identify the reference value by determining the optimal cut-off point of size related to lymph node metastasis. To validate our results, all selected patients were divided into two groups according to the cut-off point and some of the prognostic factors were compared.

Results: A moderate significant correlation was found between the tumor size and the average number of lymph node metastases $(r=0.502, P<0.01)$ and the percentage of lymph node metastasis ( $r=0.625, P<0.01$ ). The optimal cut-off reference value was $8.5 \mathrm{~mm}$ according to the receiver operating characteristic curves. Significant differences were observed for PTC prognostic factors, for example, extrathyroidal extension, multifocality, pathologic (p) N+ stage, occult metastasis in clinical (c) $\mathrm{N}$ - stage, radioactive iodine ablation, and recurrence between the two groups.

Conclusion: Due to more aggressive behavior and poorer prognosis in larger tumor size ( $>8.5 \mathrm{~mm}$ ), a tumor size $\leq 8.5 \mathrm{~mm}$ in diameter may be favorable to discriminate PTMC from PTC and aid the selection of optimal management.

Keywords: thyroid neoplasms, carcinoma, papillary, lymph nodes

\section{Introduction}

The increasing prevalence of high-resolution ultrasound and fine-needle aspiration biopsy performed under ultrasound guidance has led to remarkably and continuously increased detection and diagnosis rates of thyroid cancer over the past several decades. ${ }^{1,2}$ Papillary histologic types (from 3.4 to 12.5 per 100,000 people) have accounted for most of the registered cases ( $>80 \%$ of all thyroid tumors), with the greatest increase related to microcarcinoma. ${ }^{3,4}$ According to the World Health Organization classification, papillary thyroid carcinoma measuring $\leq 10 \mathrm{~mm}$ in greatest dimension is defined as microcarcinoma. ${ }^{5}$ Currently, theirs is the most widely used definition of papillary thyroid microcarcinoma (PTMC). However, the clinical significance of PTMC tumors $\leq 1 \mathrm{~cm}$ is widely debated; moreover, no solid basis exists for establishing the most appropriate tumor size $(\leq 1 \mathrm{~cm})$ to distinguish PTMC from papillary thyroid carcinoma (PTC), and this criterion makes the clinical risk evaluation of these lesions quite difficult. ${ }^{6}$ Importantly, as there is still no consensus regarding the optimal treatment strategy for PTMC, the 
management of PTMC ranges from observation alone to total thyroidectomy plus central lymph node dissection and postoperative radioiodine ablation therapy. ${ }^{7}$ Some investigators proposed a further subdivision of PTMC to aid optimal treatment selection, such as "micromini" 8 or "minute" for foci $5 \mathrm{~mm}$ in diameter or less. Collectively, the reason for the persistent controversy is our inability to accurately predict the aggressiveness of PTMC tumors and choose the optimal individualized management for those patients before surgery. The purpose of this article is to attempt to provide a new reference value for PTMC by determining the optimal cut-off value of size related to lymph node metastasis in PTC, which might be a preferable method to assess PTMC and assist individualized patient management decision making for these patients.

\section{Patients and methods}

We conducted a retrospective study. The medical records of 1297 patients who underwent thyroidectomy plus lymph node dissection at the Thyroid and Parathyroid Surgery Center of the West China Hospital of Sichuan University between September 2015 and July 2016 were reviewed. Of these patients, 1176 patients from 18 to 80 years of age with a histopathologic diagnosis of PTC were enrolled in this study. The exclusion criteria were other pathologic types of thyroid cancer and incomplete medical records. The data on the patients' clinical features (sex, age at diagnosis, tumor size, autoimmune thyroid disease, radioactive iodine ablation [RAI], recurrence, and reoperation) and pathologic parameters, such as extrathyroidal extension, multifocality, BRAF mutation (tested in some of the patients), pathologic (p) $\mathrm{T}$ classification and $\mathrm{p} \mathrm{N}+$ stage according to the American Joint Committee on Cancer system (version 8), were extracted from the database. All patient specimens were examined by two independent pathologists in a blind fashion. The maximum tumor size was regarded as the primary tumor size and was determined by ultrasound imaging. Receiver operating characteristic (ROC) curve studies were conducted to determine the cut-off value when the relationship of PTC tumor size and lymph node metastasis was verified by Spearman's rank correlation. Furthermore, the selected patients were divided into two groups according to the cut-off point, and factors of prognostic significance in PTC were compared, that is, extrathyroidal extension, multifocality, lymph node metastasis, adjuvant RAI, recurrence, among others. ${ }^{10-14}$ The follow-up period of the study population was 6-16 months, with a mean period of 8.7 months. Lymph node metastasis was identified not only by pathologic evidence of metastasis in the resected lymph node tissue but also by evidence of lymph node metastasis on the initial postoperative radioactive iodine imaging. ${ }^{6}$ Recurrence was defined as newly detected tumor or lymph node metastasis $>6$ months after the initial operation, as confirmed by imaging examination (mainly ultrasonography) or fine-needle aspiration biopsy. ${ }^{15}$ The institutional review board of West China Hospital approved this retrospective observational study, and written informed consent was obtained from all patients who donated their specimens.

\section{Statistical analysis}

Statistical analysis was performed using SPSS 20.0.0. Continuous variables with a normal distribution were expressed as the mean $\pm \mathrm{SD}$, and continuous variables without a normal distribution were expressed as medians. Categorical variables were presented as numbers and percentage. Spearman's rank correlation was used to analyze the relationship of tumor size and lymph node metastasis. Box plots were used to exclude extreme outliers. Continuous variables with a normal distribution were compared using Student's sample $t$-test or analysis of variance. Categorical variables were compared using the chi-square test or Fisher's exact chi-square test. A level of $5 \%$ was used to identify significant relationships.

\section{Results}

Of the 1297 patients who underwent thyroidectomy plus lymph node dissection at our institution, a pool of 1176 patients were ultimately enrolled in this retrospective study after the exclusion criteria were applied, including 355 men $(30.2 \%)$ and 821 women $(69.8 \%)$; the female/male ratio was $2.3 / 1$. The mean age was 43.7 years (range: 16-82), and the tumor size ranged from 2 to $100 \mathrm{~mm}$, with a mean tumor size of 12.8 $\mathrm{mm}$ for this group of patients. BRAF mutation positivity was observed in $240(56.2 \%)$ patients. According to the final pathology, autoimmune thyroid disease was identified in 267 (22.7\%) patients, multiple tumor formation was present in $25.3 \%$ of patients, $155(13.2 \%)$ patients appeared to have extrathyroidal extension, $682(57.9 \%)$ patients had lymph node metastasis involving only the central compartment N1a, and 226 (19.2\%) had involvement of both the central and lateral compartments N1b. During the follow-up, 547 (46.5\%) patients received radioactive iodine remnant ablation, 69 (5.9\%) patients sustained postoperative recurrence after 6 months, and 12 patients underwent reoperation. The baseline demographics and tumor characteristics are summarized in Table 1.

To determine the significance of PTC tumor size and lymph node metastasis, Spearman's rank correlation was analyzed in our study population. As shown in Figure 1, 
a moderate significant correlation was found between the tumor size and the average number of lymph node metastases $(r=0.502, P<0.01)$. Similarly, as shown in Figure 2, a significant difference was observed between the tumor size and lymph node metastasis percentage $(r=0.625, P<0.01)$; additionally, the analysis showed that a larger tumor size was associated with a significantly increased risk of lymph node metastasis and that tumor size might represent the aggressiveness of PTC to a certain extent.

Therefore, ROC curve analyses were conducted to predict the invasiveness of PTC by determining the optimal cut-off value of size related to lymph node involvement, and the

Table I Baseline demographics and tumor characteristics of the study population

\begin{tabular}{|c|c|}
\hline Variables & Value (\%) \\
\hline Age at diagnosis (mean $\pm S D$, years) & $43.7 \pm 12.9$ \\
\hline$>55$ (yes/no) & $186 / 990$ \\
\hline Sex (male/female) & $355 / 821$ \\
\hline Size of largest focus (mean $\pm S D, m m$ ) & $12.8 \pm 9.1$ \\
\hline BRAF mutation (yes/no/not applicable) & $240 / 187 / 749$ \\
\hline Autoimmune thyroid disease (yes/no) & $267 / 909$ \\
\hline Multifocality (yes/no) & $297 / 879$ \\
\hline Extrathyroidal extension (yes/no) & $155 / 1021$ \\
\hline P T classification (TI/T2/T3/T4) & $817 / 109 / 175 / 75$ \\
\hline p N stage (No/NIa/NIb) & $494 / 456 / 226$ \\
\hline P TNM stage (I/II/III/IV, AJCC version 8) & $1072 / 40 / 37 / 27$ \\
\hline RIA (yes/no) & $547 / 629$ \\
\hline Recurrence (yes/no) & $69 / 1198$ \\
\hline Reoperation (yes/no) & $12 / 1164$ \\
\hline
\end{tabular}

Abbreviations: $p$, pathologic; RIA, radioactive iodine ablation; AJCC, American Joint Committee on Cancer. evaluated optimal cut-off point of tumor size in this study was $8.5 \mathrm{~mm}$ (area under the curve: 0.723 ) in Figure 3. Importantly, we considered that the tumor sizes of the enrolled PTC patients in this study, which included extreme values such as $100 \mathrm{~mm}$, may have impacted the optimal cut-off point. As shown in Figures 4 and 5, we employed a box plot method to exclude extreme outliers and performed ROC curve analysis again in patients with tumor sizes between 2 and $40 \mathrm{~mm}$, which unexpectedly showed the same result: the optimal calculated cut-off point was $8.5 \mathrm{~mm}$ (area under the curve: 0.706). In conclusion, the results suggested that tumors with a diameter $>8.5 \mathrm{~mm}$ had a stronger predilection for lymph node metastasis than those with a diameter $\leq 8.5 \mathrm{~mm}$.

Additionally, to verify the rationality of the cut-off point, all the patients were divided into two groups according to the tumor size using the cut-off point (Group A and Group B), as shown in Table 2. Of these patients, 445 and 731 patients belonged to Group A ( $\leq 8.5 \mathrm{~mm})$ and Group B $(>8.5 \mathrm{~mm})$, respectively. Next, the essential prognostic factors were compared. No significant differences were found for age, the presence of autoimmune thyroid disease, BRAF mutation, and reoperation between the two groups (all $P>0.5$ ). The cut-off point of tumor size, however, was significantly related to the male sex and the risk of extrathyroidal extension, multifocality, lymph node metastasis, RAI, and recurrence (all $P<0.05)$. Among the patients, males comprised $236(32.3 \%)$ patients in Group B compared with 119 (26.7\%) patients in Group A. The patients in Group B showed higher rates of

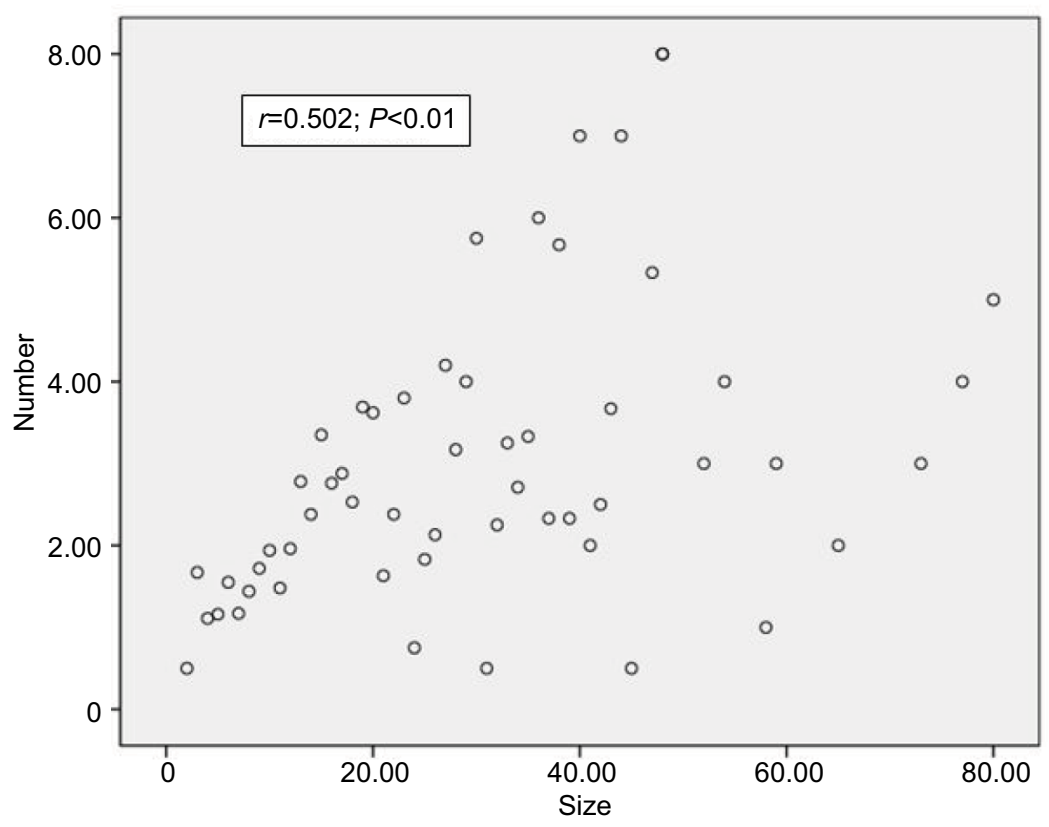

Figure I Spearman's correlation analysis between tumor size and average number of lymph node metastases. 


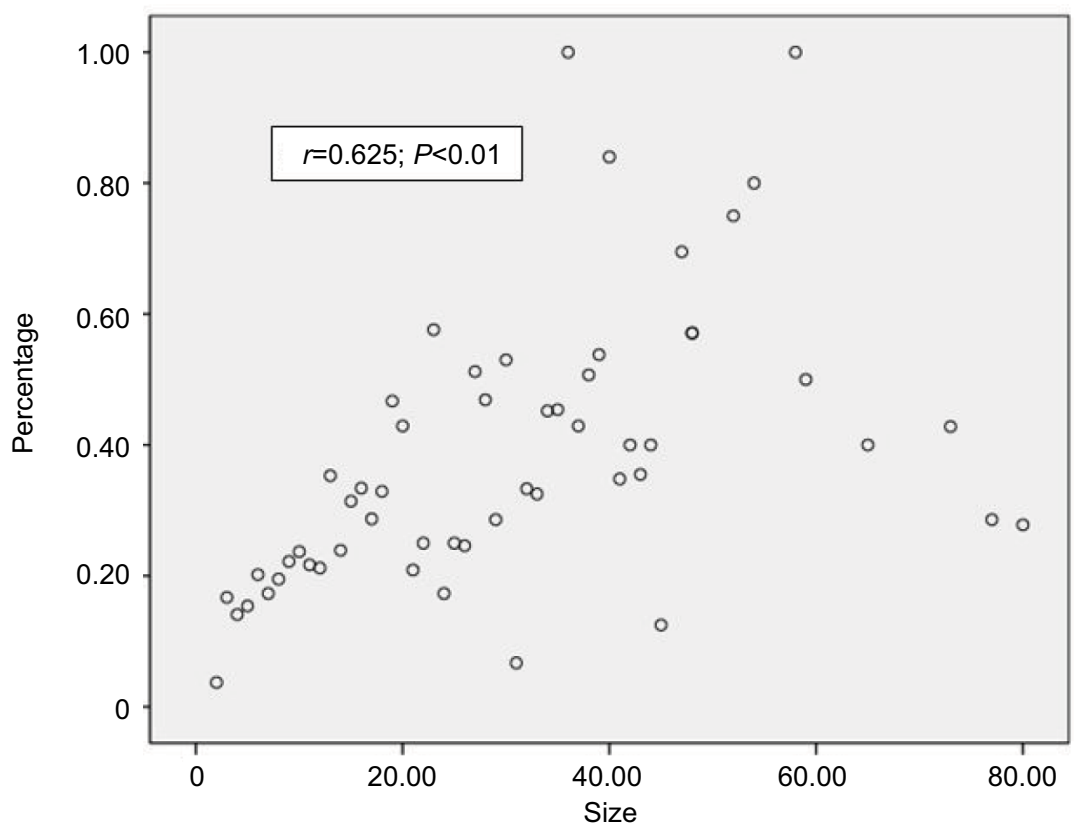

Figure 2 Spearman's correlation analysis between tumor size and lymph node metastasis percentage.

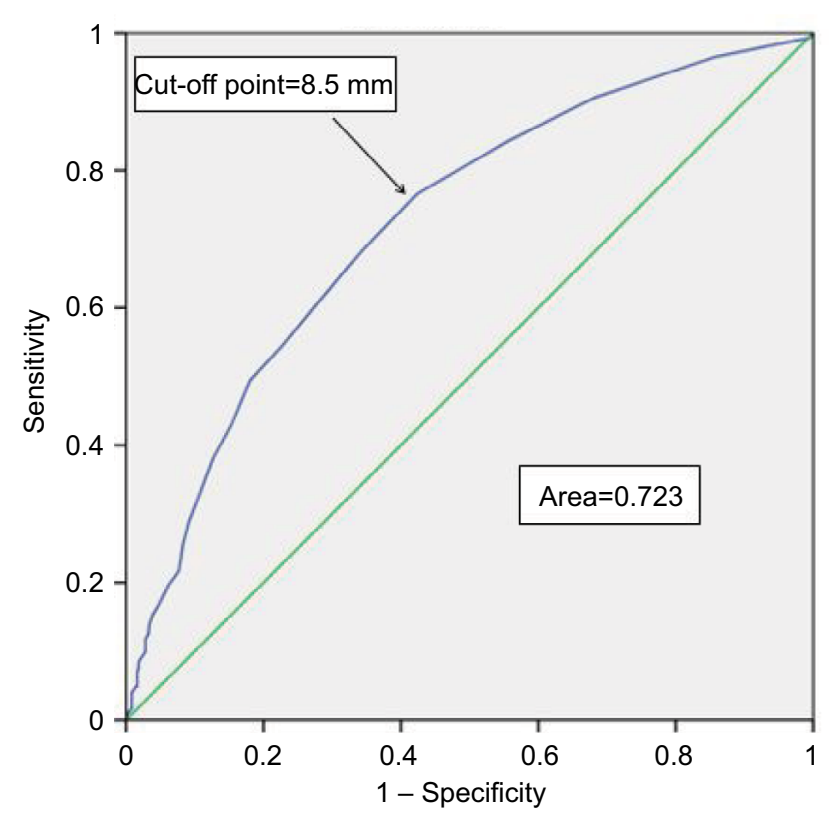

Figure 3 ROC curve to predict the risk of lymph node metastasis. Abbreviation: ROC, receiver operating characteristic.

multifocality $(27.8 \%$ vs $21.1 \%)$, extrathyroidal extension (14.9\% vs $10.3 \%)$, p $\mathrm{N}+$ stage $(71.4 \%$ vs $36.0 \%)$, occult metastasis in clinical (c) $\mathrm{N}-$ stage $(56.5 \%$ vs $25.4 \%)$, RAI (51.2\% vs $38.9 \%)$, and recurrence (7.1\% vs $3.8 \%)$.

\section{Discussion}

Neck ultrasonography combined with fine-needle aspiration cytology has led to dramatically increased registered cases of thyroid carcinoma recently. Specifically, since 1975, the incidence of thyroid cancer has nearly tripled from 4.9 to 14.3 per 100,000 people $^{3}$ and the particular variant of PTMC accounts for up to $30 \%$ of all the differentiated thyroid carcinoma cases. ${ }^{16}$ These increased microcarcinoma cases account for $38.5 \%$ of the papillary thyroid cancers in the US, up to $48.8 \%$ in France, and 35.7\% in Shanghai, China. ${ }^{17-21}$ However, many current guidelines and recommendations regarding the optimal treatment strategy for PTMC remain controversial. Considerable debate has centered on the clinical significance of PTMC and whether these tumors should be managed as aggressively as other malignancies of the thyroid. Although PTMC is considered an indolent cancer with relatively benign biologic behavior and an excellent prognosis, certain aspects of PTMC biologic behavior are quite poor, which may present as lymph node metastasis, distant metastasis, recurrence, and may even cause death. ${ }^{22-24}$ As the reported mortality rate of PTMC ranges from $0 \%$ to $1 \%$ and presents relatively benign biologic behavior, ${ }^{8}$ some researchers reported that PTMC should not be overtreated. Rather, periodic observation without surgical treatment ${ }^{1}$ has been suggested, and in the American Thyroid Association and the British Thyroid Association guidelines, lobectomy was described as the primary operative management of PTMC without aggressive features, for example, extrathyroidal extension, multifocality, and central or lateral lymph node metastasis, ${ }^{25,26}$ moreover, prophylactic central lymph node dissection was not recommended without a suspicious enlarged lymph node in the treatment of PTC $^{27}$ 


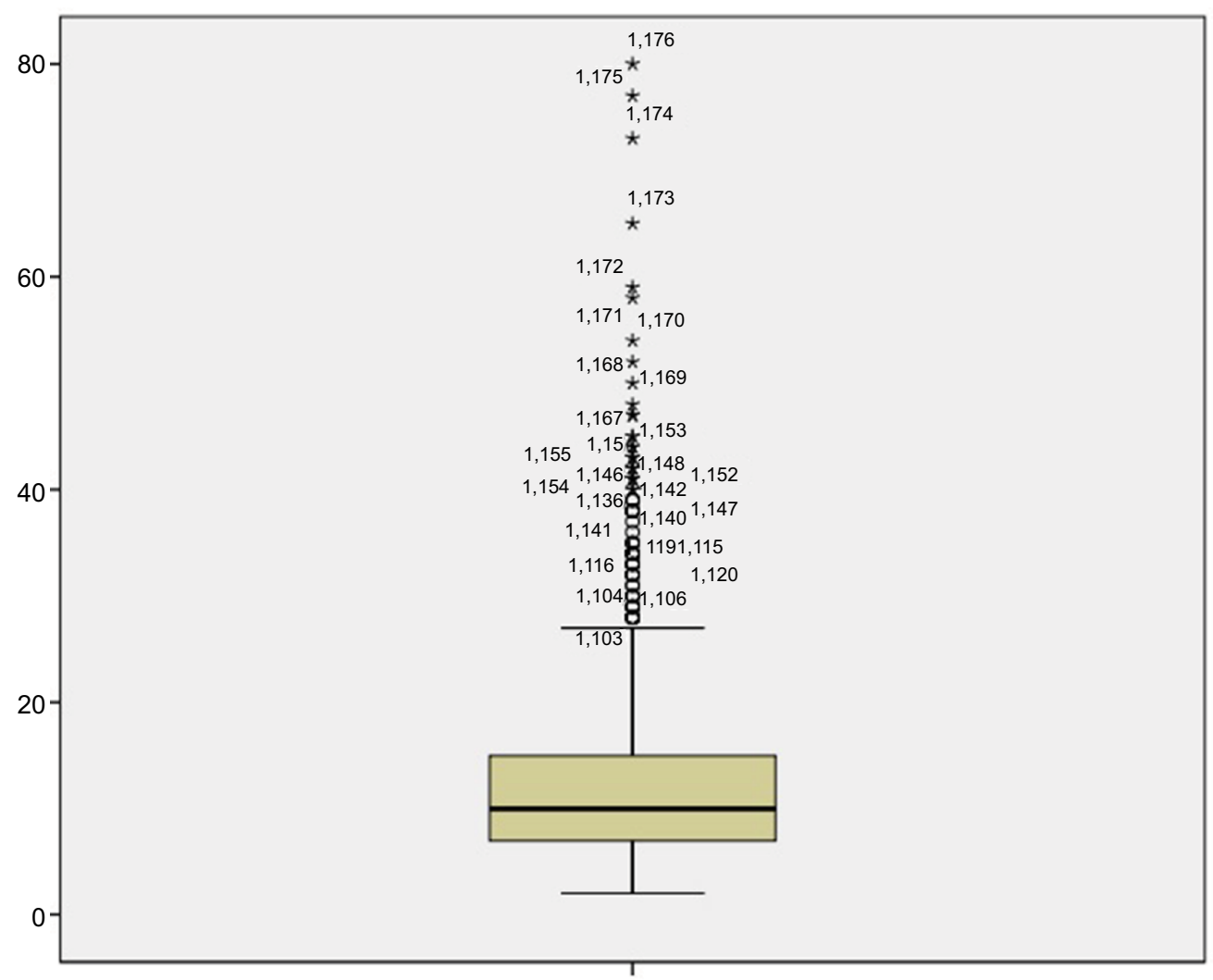

Figure 4 The outliers of tumor size in the study population.

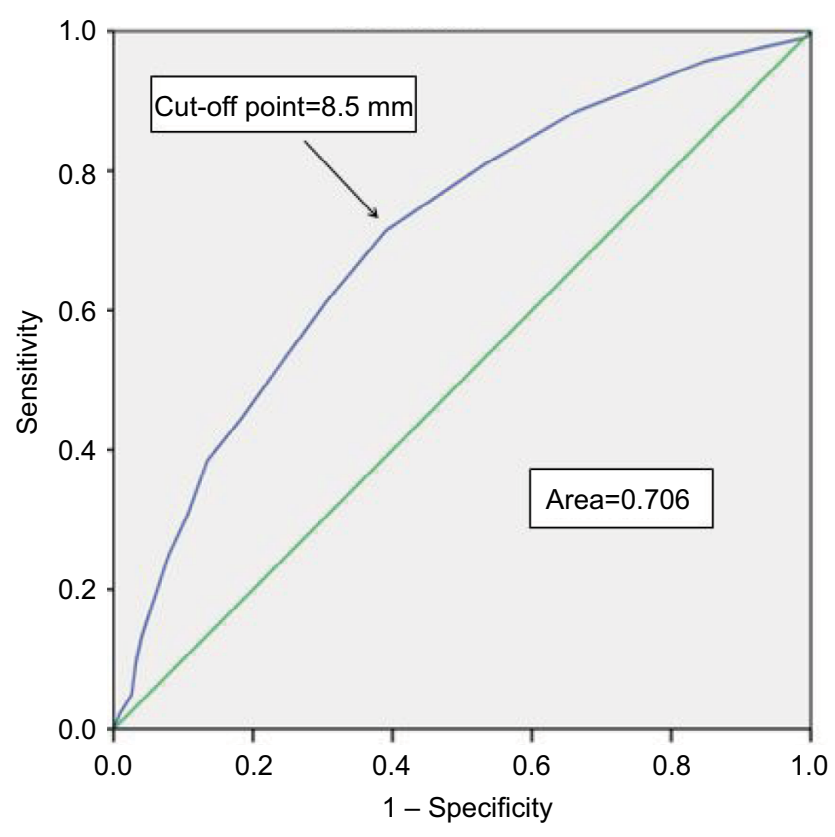

Figure 5 ROC curve to predict the risk of lymph node metastasis in PTC with tumor sizes between 2 and $40 \mathrm{~mm}$.

Abbreviation: ROC, receiver operating characteristic. because it provides no survival benefit ${ }^{28}$ and may even increase the incidence of postoperative complications. ${ }^{29}$ In Conzo et al's study, patients underwent total thyroidectomy with increasing rate of transitory or definitive hypoparathyroidism, laryngeal nerve injury, and cervical hematoma. ${ }^{30}$ However, other studies demonstrated that total or near-total thyroidectomy is associated with reduced recurrence rates compared with lobectomy; moreover, completion thyroidectomy is relatively safe in the hands of an experienced surgeon. ${ }^{31}$ Finally, total or near-total thyroidectomy offers treated patients a chance to receive radioactive iodine therapy postoperatively, which has been shown to improve survival and reduce recurrence; ${ }^{32,33}$ additionally, it also offers the possibility of better surveillance by aiming to detect persistent disease or recurrence by thyroglobulin measurements and scintigraphic scans. Central lymph node dissection permits accurate assessment of the tumor stage postoperatively. ${ }^{34}$ Furthermore, the risk of recurrence is significantly increased in PTMC patients with central lymph node metastasis and a second operation will increase the risk of postoperative complications. . $^{35,36}$ 
Table 2 Analysis of clinical and pathologic characteristics of PTC divided by cut-off value of tumor size

\begin{tabular}{|c|c|c|c|}
\hline \multirow[t]{2}{*}{ Variables } & \multirow{2}{*}{$\begin{array}{l}\text { Group A }(\leq 8.5 \mathrm{~mm}) \\
\mathrm{n}=445\end{array}$} & \multirow{2}{*}{$\begin{array}{l}\text { Group B (>8.5 mm) } \\
\mathrm{n}=73 \mathrm{I}\end{array}$} & \multirow[t]{2}{*}{$P$-value } \\
\hline & & & \\
\hline Age at diagnosis (mean $\pm \mathrm{SD}$, years) & $44.6 \pm 13.1$ & $43.1 \pm 12.8$ & \\
\hline$>55$ (yes $/$ no $)$ & $65 / 380$ & $121 / 610$ & 0.375 \\
\hline Sex (male/female) & $119 / 326$ & $236 / 495$ & $0.045^{*}$ \\
\hline Autoimmune thyroid disease (yes/no) & $101 / 344$ & $166 / 565$ & 0.996 \\
\hline Multifocality (yes/no) & $94 / 351$ & $203 / 528$ & $0.011^{*}$ \\
\hline Extrathyroidal extension (yes/no) & $46 / 399$ & $109 / 622$ & $0.025^{*}$ \\
\hline p N stage $(\mathrm{N} 0 / \mathrm{NIa} / \mathrm{NIb})$ & $285 / 132 / 28$ & $209 / 324 / 198$ & $<0.01 *$ \\
\hline Occult metastasis in c N- stage (yes/no) & $97 / 285$ & $272 / 209$ & $<0.0 I^{*}$ \\
\hline BRAF mutation (yes/no/not applicable) & $101 / 88 / 256$ & $139 / 99 / 493$ & 0.304 \\
\hline RIA (yes/no) & $173 / 272$ & $374 / 357$ & $<0.0 I^{*}$ \\
\hline Recurrence (yes/no) & $17 / 428$ & $52 / 679$ & $0.019 *$ \\
\hline Reoperation (yes/no) & $5 / 440$ & $7 / 724$ & 0.776 \\
\hline
\end{tabular}

Note: *Statistically significant differences.

Abbreviations: c, clinical; p, pathologic; PTC, papillary thyroid carcinoma; RIA, radioactive iodine ablation.

Malignant tumors, being distinct from benign, harbor aggressive features. Before treatment, an accurate predictive assessment of the tumor aggressiveness will aid the physician in choosing the optimal individualized patient management. Because PTMC is being diagnosed with increasing frequency, it is important to describe the clinical and histologic characteristics that confer cancer aggressiveness. It is quite likely that molecular studies will be able to discriminate aggressive PTMC from the tumors with an indolent clinical course and to determine whether any specific morphologic and/or molecular marker exists that predicts the clinical behavior and distinguishes PTMC from PTC. However, the size of the tumor is widely used to predict the aggressiveness of PTMC to aid clinical decision making because it is the easiest parameter to determine using ultrasound images. In previous studies, investigators have attempted to identify the tumor diameter cut-off value predictive of aggressiveness by ROC curves in PTMC (tumor size $\leq 10 \mathrm{~mm}$ ) and have proposed a further subdivision of PTMC by cut-off point related to lymph node metastasis to guide management of these minute lesions. ${ }^{89}$ This proposal has had highly variable results, with some studies reporting that tumors $\leq 5 \mathrm{~mm}$ are less likely to metastasize, while other studies have reported a cut-off of 6,7 , or $8 \mathrm{~mm} .{ }^{37-39}$

To the best of our knowledge, this study may be a novel report that may assist the selection of optimal management for these minute lesions by focusing on the definition of PTMC. In this study, a moderate significant correlation was found between the tumor size and the average number of lymph node metastases $(r=0.502, P<0.01)$ and the lymph node metastasis percentage $(r=0.625, P<0.01)$. The optimal cut-off value of size related to lymph node metastasis was $8.5 \mathrm{~mm}$ in PTC patients, regardless of whether box plots were used to exclude extreme outliers by ROC curves (areas under the curve: 0.723 and 0.706 , respectively). We then divided the patients into two groups by the tumor diameter $(8.5 \mathrm{~mm})$ and compared many prognostic factors between the two groups. Although a small gap was observed in the two groups in terms of male sex (32.3\% vs $26.7 \%$ ), extrathyroid extension ( $14.9 \%$ vs $10.3 \%)$, and multifocality ( $27.8 \%$ vs $21.1 \%)$, the significant statistical difference was found due to relative large scale in our present study. Similarly, significant differences were also observed for $\mathrm{p} \mathrm{N}+$ stage, occult metastasis in c N- stage, RAI, and recurrence between the two groups. In Roti et al's study of PTMC, ${ }^{39}$ patients with tumor size $\geq 8 \mathrm{~mm}$ had higher cancer aggressiveness, approximately consistent with our current study, which suggested that tumors with a diameter $>8.5 \mathrm{~mm}$ might be more aggressive and have a worse prognosis than those with a diameter $\leq 8.5 \mathrm{~mm}$. Therefore, a tumor size $\leq 8.5 \mathrm{~mm}$ in diameter may be a new reference value for microcarcinoma. During follow-up, we observed that 69 patients (5.8\%) had recurrences (17 cases in Group A and 52 cases in Group B) and none experienced cancer-related mortality. Because of the limited follow-up (6-16 months), the results concerning the recurrence rate are likely underestimated; thus, recurrence-free survival curves between the two groups were not shown in this study. However, we will follow-up these patients continually and construct disease-free survival curves to further determine the clinical significance of the aforementioned findings in the future.

Taken together, the size of the tumor determined by ultrasound images was related to the risk of lymph node metastasis in PTC. According to a new reference value identified by the ROC curves, a tumor size $\leq 8.5 \mathrm{~mm}$ in diameter may be a favorable tumor size to distinguish PTMC from PTC, and it could be an easy predictor for the clinical significance of PTC and assist us to choose optimal treatment. 


\section{Acknowledgments}

This study was supported by grants from National Key R\&D Program of China (2017YF0907504), National Natural Science Foundation (81702646), Sichuan Province Science and Technology Project of China (No. 2017SZ0139), Sichuan University for youth fund (2017SCU11016), Health and Family Planning Commission of Sichuan Province (17PJ398), and Postdoctoral Sustentation Fund of Sichuan University (2017SCU12035). YPG, GPL, and JYL contributed equally to this study and are co-first authors.

\section{Author contributions}

All authors made substantial contributions to conception and design, acquisition of data, or analysis and interpretation of data; took part in drafting the article or revising it critically for important intellectual content; gave final approval of the version to be published; and agree to be accountable for all aspects of the work. JQZ is the guarantor. YPG, GPL and JYL contributed equally to this work and are co-first authors.

\section{Disclosure}

The authors report no conflicts of interest in this work.

\section{References}

1. Ito Y, Uruno T, Nakano K, et al. An observation trial without surgical treatment in patients with papillary microcarcinoma of the thyroid. Thyroid. 2003;13(4):381-387.

2. Yokozawa T, Fukata S, Kuma K, et al. Thyroid cancer detected by ultrasound-guided fine-needle aspiration biopsy. World J Surg. 1996;20(7): 848-853.

3. Wells JS, Holstad MM, Thomas T, et al. Increased incidence of thyroid cancer associated with increased diagnosis. J Calif Dent Assoc. 2014;42(4):220.

4. Chen AY, Jemal A, Ward EM. Increasing incidence of differentiated thyroid cancer in the United States, 1988-2005. Cancer. 2009;115(16) 3801-3807.

5. Sobin LH. Histological typing of thyroid tumours. Histopathology. 1990;16(5):513

6. Arora N, Turbedian HK, Kato MA, Moo TA, Zarnegar R, Fahey TJ Papillary thyroid carcinoma and microcarcinoma: is there a need to distinguish the two? Thyroid. 2009;19(5):473-477.

7. Wu AW, Nguyen C, Wang MB. What is the best treatment for papillary thyroid microcarcinoma? Laryngoscope. 2011;121(9):1828-1829.

8. Wang M, Wu WD, Chen GM, et al. Could tumor size be a predictor for papillary thyroid microcarcinoma: a Retrospective Cohort Study. Asian Pac J Cancer Prev. 2015;16(18):8625-8628.

9. Kasai N, Sakamoto A. New subgrouping of small thyroid carcinomas. Cancer. 1987;60(8):1767-1770.

10. Yamamoto Y, Maeta T, Izumi K, Otsuka H. Occult papillary carcinoma of the thyroid. A study of 408 autopsy cases. Cancer. 1990;65(5): 1173-1179.

11. Strate SM, Lee EL, Childers JH. Occult papillary carcinoma of the thyroid with distant metastases. Cancer. 1984;54(6):1093-1100.

12. Pellegriti G, Scollo C, Lumera G, Regalbuto C, Vigneri R, Belfiore A. Clinical behavior and outcome of papillary thyroid cancers smaller than $1.5 \mathrm{~cm}$ in diameter: study of 299 cases. J Clin Endocrinol Metab. 2004;89(8):3713-3720.
13. Cady B. Primary carcinoma of the thyroid. Sem Surg Oncol. 1991;7(3):81-86.

14. Tsuchiya A, Suzuki S, Kanno M, Kikuchi Y, Ando Y, Abe R. Prognostic factors associated with differentiated thyroid cancer. Surg Today. 1995;25(9):778-782.

15. Kim SK, Park I, Woo JW, et al. Total thyroidectomy versus lobectomy in conventional papillary thyroid microcarcinoma: analysis of 8,676 patients at a single institution. Surgery. 2017;161(2):485-492.

16. Ito $\mathrm{Y}$, Tomoda $\mathrm{C}$, Uruno $\mathrm{T}$, et al. Papillary microcarcinoma of the thyroid: how should it be treated? World J Surg. 2004;28(11):1115-1121.

17. Giordano D, Gradoni P, Oretti G, Molina E, Ferri T. Treatment and prognostic factors of papillary thyroid microcarcinoma. Clin Otolaryngol. 2010;35(2):118-124.

18. Kutler DI, Crummey AD and Kuhel WI. Routine central compartment lymph node dissection for patients with papillary thyroid carcinoma. Head Neck. 2012;34(2):260-263.

19. Garrel R, Tripodi C, Cartier C, Makeieff M, Crampette L, Guerrier B. Cervical lymphadenopathies signaling thyroid microcarcinoma. Case study and review of the literature. Eur Ann Otorhinolaryngol Head Neck Dis. 2011;128(3):115-119.

20. Zhao Q, Ming J, Liu C, et al. Multifocality and total tumor diameter predict central neck lymph node metastases in papillary thyroid microcarcinoma. Ann Surg Oncol. 2013;20(3):746-752.

21. Xiang J, Wu Y, Li DS, et al. New clinical features of thyroid cancer in eastern China. J Visc Surg. 2010;147(1):e53-e56.

22. Zafon C, Baena JA, Castellví J, Obiols G, Monroy G, Mesa J. Differences in the form of presentation between papillary microcarcinomas and papillary carcinomas of larger size. J Thyroid Res. 2010;2011: 639156.

23. Ito $\mathrm{Y}$, Tomoda $\mathrm{C}$, Uruno $\mathrm{T}$, et al. Papillary microcarcinoma of the thyroid: how should it be treated? World J Surg. 2004;28(11):1115-1121.

24. Marcy PY, Thariat J, Peyrottes I, et al. Fulminant lethal spread of occult papillary microcarcinoma of the thyroid. Thyroid. 2010;20(4): 445-448.

25. American Thyroid Association (ATA) Guidelines Taskforce on Thyroid Nodules and Differentiated Thyroid Cancer, Cooper DS, Doherty GM, Haugen BR, et al. Revised American Thyroid Association management guidelines for patients with thyroid nodules and differentiated thyroid cancer. Thyroid. 2009;19(11):1167-1214.

26. Perros P, Boelaert K, Colley S, et al; British Thyroid Association. Guidelines for the management of thyroid cancer. Clin Endocrinol (Oxf). 2014;81(Suppl 1):1-122.

27. Gambardella C, Tartaglia E, Nunziata A, et al. Clinical significance of prophylactic central compartment neck dissection in the treatment of clinically node-negative papillary thyroid cancer patients. World J Surg Oncol. 2016;14(1):247.

28. Appetecchia M, Scarcello G, Pucci E, Procaccini A. Outcome after treatment of papillary thyroid microcarcinoma. J Exp Clin Cancer Res. 2002;21(2):159-164.

29. Wada N, Duh QY, Sugino K, et al. Lymph node metastasis from 259 papillary thyroid microcarcinomas: frequency, pattern of occurrence and recurrence, and optimal strategy for neck dissection. Ann Surg. 2003,237(3):399-407.

30. Conzo G, Avenia N, Ansaldo GL, et al. Surgical treatment of thyroid follicular neoplasms: results of a retrospective analysis of a large clinical series. Endocrine. 2016;55(2):530-538.

31. Kupferman ME, Mandel SJ, DiDonato L, Wolf P, Weber RS. Safety of completion thyroidectomy following unilateral lobectomy for well-differentiated thyroid cancer. Laryngoscope. 2002;112(7 Pt 1): 1209-1212.

32. Mazzaferri EL, Jhiang SM. Long-term impact of initial surgical and medical therapy on papillary and follicular thyroid cancer. $\mathrm{Am} \mathrm{J} \mathrm{Med.}$ 1994;97(5):418-428.

33. Samaan NA, Schultz PN, Hickey RC, et al. Well-differentiated thyroid carcinoma and the results of various modalities of treatment. A retrospective review of 1599 patients. J Clin Endocrinol Metab. 1992;75(3) 714-720. 
34. Levin KE, Clark AH, Duh QY, Demeure M, Siperstein AE, Clark OH. Reoperative thyroid surgery. Surgery. 1992;111(6): 604-609.

35. Chow SM, Law SC, Chan JK, Au SK, Yau S, Lau WH. Papillary microcarcinoma of the thyroid-prognostic significance of lymph node metastasis and multifocality. Cancer. 2003;98(1):31-40.

36. Vini L, Hyer SL, Marshall J, A'Hern R, Harmer C. Long-term results in elderly patients with differentiated thyroid carcinoma. Cancer. 2003;97(11):2736-2742.
37. Lim YC, Choi EC, Yoon YH, Kim EH, Koo BS. Central lymph node metastases in unilateral papillary thyroid microcarcinoma. Br J Surg. 2009;96(3):253-257.

38. Zhang L, Wei WJ, Ji QH, et al. Risk factors for neck nodal metastasis in papillary thyroid microcarcinoma: a study of 1066 patients. $J$ Clin Endocrinol Metab. 2012;97(4):1250-1257.

39. Roti E, Rossi R, Trasforini G, et al. Clinical and histological characteristics of papillary thyroid microcarcinoma: results of a retrospective study in 243 patients. J Clin Endocrinol Metab. 2006;91(6):2171-2178.

\section{Publish your work in this journal}

Cancer Management and Research is an international, peer-reviewed open access journal focusing on cancer research and the optimal use of preventative and integrated treatment interventions to achieve improved outcomes, enhanced survival and quality of life for the cancer patient. The manuscript management system is completely online and includes a very quick and fair peer-review system, which is all easy to use. Visit http://www.dovepress.com/testimonials.php to read real quotes from published authors. 